\title{
Modern sands of the Gulf of Mexico: Discriminating fluvial and coastal sand composition
}

\section{Arenas actuales del Golfo de México: Discriminación entre la composición de arenas fluviales y costeras}

\author{
J.J. Kasper-Zubillaga* \\ A. Carranza-Edwards \\ Instituto de Ciencias del Mar y Limnología \\ UNAM \\ Circuito Exterior $\mathrm{s} / \mathrm{n}, \mathrm{Cd}$. Universitaria \\ Coyoacán, 04510, México D.F., México \\ *E-mail: kasper@icmyl.unam.mx
}

Recibido en marzo de 2003; aceptado en julio de 2003

\begin{abstract}
A forward stepwise linear discriminant analysis, A-Nova test and R-mode factor analysis were used on modern beach, dune and river sands to discriminate the geographic distribution of detrital modes between sands sourced by volcanic rocks (Trans-Mexican Volcanic Belt (TMVB) sands) and sedimentary rocks (Veracruz Embayment (VE) sands). Thirty-three beach, dune and river sand samples were collected across beach-dune profiles, in the inner reaches of the channel and at the river mouth during the dry season. The source terranes for these sands are constituted by Miocene to Pliocene volcanites and by Tertiary to Quaternary siliciclastic rocks. To avoid the constrained character of compositional data, point-count percentages were expressed as compositional modal $\log$ ratios (i.e., $\log \mathrm{Qt} / \mathrm{Ft}=$ total quartz/total feldspars, $\log \mathrm{Qt} / \mathrm{Lt}=$ total quartz/total lithics, $\log \mathrm{Ft} / \mathrm{Lt}=$ total feldspars/total lithics, Log $\mathrm{Qm} / \mathrm{Fk}=$ monocrystalline quartz/potash feldspars, Log $\mathrm{Qm} / \mathrm{Fp}=$ monocrystalline quartz/ plagioclase, $\log \mathrm{Fk} / \mathrm{Fp}=$ potash feldspars/plagioclase, and $\mathrm{Log} \mathrm{Lv} / \mathrm{Ls}=$ volcanic lithics/sedimentary lithics). The linear discriminant and factor analyses showed that the geographical dispersal of detrital modes between the TMVB sands and VE sands are statistically significant by means of the $\log \mathrm{Lv} / \mathrm{Ls}, \log \mathrm{Qt} / \mathrm{Ft}, \log \mathrm{Qt} / \mathrm{Lt}$ and $\log \mathrm{Ft} / \mathrm{Lt}$ ratios. The A-Nova test and post-hoc analyses showed that the mean differences of the Log Lv/Ls, Log Qt/Ft and Log Qt/Lt ratios are highly significant for the VE river sands and the TMVB dune sands. The composition of the TMVB dune sands is controlled by a longshore drift of quartz supply. The VE river sands are depleted in Ls due to the mechanical and/or chemical weathering of Ls and the influence of TMVB on the composition of the VE river sands.
\end{abstract}

Key words: modern and detrital modes, linear discriminant analysis, A-Nova test, factor analysis, SW Gulf of Mexico.

\section{Resumen}

Se realizó un análisis de discriminación lineal, una prueba A-Nova y un análisis factorial en arenas actuales de playa, duna y río para discriminar la distribución geográfica composicional entre arenas derivadas de fuentes volcánicas (arenas del Cinturón Volcánico Trans-Mexicano, CVTM) y sedimentarias (arenas de la Cuenca Veracruzana, CV). Se recolectaron 33 muestras de arena de playa, duna y río a lo largo de perfiles de playa-duna, en la desembocadura y el lecho distal de los ríos en la época de secas. La roca fuente está constituida por rocas volcánicas del Mioceno y Plioceno y rocas silicoclásticas del Terciario al Cuaternario. Para evitar el carácter "cerrado" de los datos composicionales, los porcentajes obtenidos del conteo de granos se expresaron como índices logarítmicos $(\log \mathrm{Ct} / \mathrm{Ft}=$ cuarzo total/feldespato total, $\log \mathrm{Ct} / \mathrm{Lt}=$ cuarzo total/líticos totales, $\log \mathrm{Ft} / \mathrm{Lt}=$ feldespato total/líticos totales, $\log \mathrm{Cm} / \mathrm{Fk}=$ cuarzo monocristalino/feldespato de potasio, $\log \mathrm{Cm} / \mathrm{Fp}=$ cuarzo monocristalino/plagioclasas, $\log \mathrm{Fk} / \mathrm{Fp}=$ feldespato de potasio/plagioclasas y $\mathrm{Log} \mathrm{Lv} / \mathrm{Ls}=$ líticos volcánicos/líticos sedimentarios). El análisis de discriminación lineal y el análisis factorial mostraron que las diferencias geográficas composicionales de la arena de playa, duna y río del CVTM y de la CV son estadísticamente significativas mediante los índices $\log \mathrm{Lv} / \mathrm{Ls}$, $\log \mathrm{Ct} / \mathrm{Ft}$, Log Ct/Lt y Log Ft/Lt. Más aún, las pruebas A-Nova y post-hoc mostraron diferencias significativas en las medias de los índices Log Lv/Ls, Log Ct/Ft y Log Ct/Lt para la arena de duna del CVTM y de la arena de río de la CV. La composición de la arena de duna del CVTM está influída por transporte de cuarzo a lo largo de la costa. La ausencia de Ls en la composición de la arena de río de la CV está determinada por el intemperismo mecanico y/o químico de los Ls y la influencia del CVTM en la composición de la arena de río de la CV.

Palabras clave: arenas actuales y procedencia, análisis de discriminación lineal, prueba A-Nova, análisis factorial, Golfo de México sur occidental. 


\section{Introduction}

Sand detrital modes are controlled by the source-rock composition, climate, relief and drainage system (Basu, 1976; Ibbeken and Schleyer, 1991; Le Pera and Critelli, 1997). Beach and dune sands reflect provenance from continental sources, and are controlled by longshore transport and fluvial discharges (Komar, 1976; Critelli et al., 1997). The spatial distribution of beach, dune and river sand composition, however, has not yet been tested using multivariate analyses. Moreover, multivariate methods such as A-Nova tests and post-hoc group comparisons have not been included together with the linear discriminant analysis (LDA) and factor analysis (FA) to test for significant variables and group differences. The purpose of this study is to detect the spatial distribution of the sand modes during the dry season in order to assess the feasibility of such an approach for the characterization of sand provenance and discrimination among sand composition environments of the area by means of LDA, A-Nova and FA.

\section{Study area}

The area is located in the southwestern coastal Gulf of Mexico, at $19^{\circ} 25^{\prime}-19^{\circ} 48^{\prime} \mathrm{N}$ latitude and $96^{\circ} 20^{\prime}-96^{\circ} 27^{\prime} \mathrm{W}$ longitude (fig. 1). In the Tampico Embayment (TE) and Veracruz Embayment (VE), the relief corresponds to plains, while in the Trans-Mexican Volcanic Belt (TMVB), the mountainous relief reaches the coastline (Lugo-Hubp et al., 1990). In figure 2 we observe how the altitude ( 200 and $1000 \mathrm{~m}$ ) contour lines delineate these geomorphologic embayments. The terrain gradient is maximum in the TMVB sand zone (cf. slope 0.09500), and gradually decreases towards the north (cf. slope 0.00704 ) and south (cf. slope 0.00487). Across the VE sand zone, the average gradient is 0.01900 . Between the mountainous relief and the plains there is an area of hills. In the TMVB sand zone, torrential streams dominate, whereas the VE sands are directly affected by rivers that originate in lands higher than $1000 \mathrm{~m}$ in the mountainous relief areas with high precipitation (sometimes higher than $4000 \mathrm{~mm}$ ) (fig. 3). In the study area, the rainy season is from June to October and the dry season from November to May (CETENAL, 1970), with a dominant humid semi-tropical climate. The area is a wave-dominated zone (waves $0.9 \mathrm{~m}$ in height), influenced by surface water currents with a southward component in spring and average velocities of $6 \mathrm{~cm} \mathrm{~s}^{-1}$ (Fernández-Eguiarte et al., 1992a, b). The study area is drained by torrential streams close to TMVB and by two rivers with a perennial regime in VE (figs. 1,2). The area is the extension of the TMVB (Negendank et al., 1985), characterized by Miocene-Pliocene andesites of calcalkaline composition (Cantagrel and Robin, 1979; Negendank et al., 1985) and alkaline basalts (Negendank et al., 1985) (fig. 1) inferred to reflect the Cocos Plate subduction. The TE and VE are sedimentary deposits composed of Tertiary and Quaternary alluvial strata of siliciclastic and minor carbonate composition (fig. 1).

\section{Introducción}

Las modas detríticas de las arenas son controladas por la composición de la roca fuente, el clima, el relieve y el sistema de drenaje de la cuenca (Basu, 1976; Ibbeken y Schleyer, 1991; Le Pera y Critelli, 1997). Las arenas de playa y de duna reflejan su proveniencia de fuentes continentales, y son influidas por el transporte costero y las descargas fluviales (Komar, 1976; Vritelli et al., 1997). La distribución espacial de la composición de arenas de playa, duna y río, sin embargo, no ha sido aún probada por medio de análisis multivariado. Tampoco se han incluido métodos multivariados tales como el A-Nova y las comparaciones de grupos post-hoc junto con análisis de discriminación lineal (ADL) y análisis factorial (AF) para probar la significancia de variables y diferencias de grupos. El propósito de este estudio es detectar la distribución espacial de las modas de arena durante la temporada de secas a fin de evaluar la factibilidad de los métodos de ADL, A-Nova y AF para caracterizar la proveniencia y discriminar entre ambientes composicionales de las arenas.

\section{Área de estudio}

El área de estudio se encuentra en la costa sur occidental del Golfo de México entre $19^{\circ} 25^{\prime}-19^{\circ} 48^{\prime} \mathrm{N}$ y $96^{\circ} 20^{\prime}-96^{\circ} 27^{\prime} \mathrm{W}$ (fig. 1). El relieve de la Cuenca Veracruzana (CV) y la Cuenca de Tampico (CT) corresponde a planicies, mientras que en el Cinturón Volcánico Trans-Mexicano (CVTM) el relieve montañoso llega hasta la línea de costa (Lugo-Hubp et al., 1990). En la figura 2 se observa cómo las cotas de nivel (200 y $1000 \mathrm{~m}$ ) delinean estas cuencas geomorfológicas. Existe un gradiente máximo del terreno en la zona de arenas del CVTM (pendiente cf. 0.09500) que disminuye gradualmente hacia el norte (pendiente cf. 0.00704) y el sur (cf. 0.00487). El gradiente medio de la zona de arenas de la CV es 0.01900. Entre el relieve montañoso y las planicies existe una zona de colinas. En la zona de arenas del CVTM dominan los arroyos intermitentes mientras que las arenas de la $\mathrm{CV}$ se ven directamente afectadas por ríos que se originan en tierras por encima de los $1000 \mathrm{~m}$ de altitud, en las áreas de relieve montañoso con altos niveles de precipitación (en ocasiones mayores a $4000 \mathrm{~mm}$ ) (fig. 3). En el área de estudio se presenta un clima dominante semi-tropical húmedo con la temporada de lluvias de junio a octubre y la de secas de noviembre a mayo (CETENAL, 1970). El área está dominada por el oleaje (olas de $0.9 \mathrm{~m}$ de altura), influenciada por corrientes de agua superficiales con una componente en dirección sur en primavera y velocidades medias de $6 \mathrm{~cm} \mathrm{~s}^{-1}$ (Fernández-Eguiarte et al., 1992a, b). Cerca del CVTM el área de estudio es drenada por arroyos intermitentes y en la $\mathrm{CV}$ el drenaje es por dos ríos de régimen perenne (figs. 1, 2). El área es la extensión del CVTM (Negendank et al., 1975) que se caracteriza por andesitas de composición calcicoalcalina (Cantagrel y Robin, 1979; Negendank et al., 1985) y basaltos alcalinos del Mioceno-Plioceno (Negendank et al., 1985) (fig. 1) que se suponen un reflejo de la subducción de la 


\section{Materials and methods}

A total of 33 sediment samples were collected. Sand sampling was carried out only during the dry season where the VE river sands are still draining towards the sea (fig. 1). Petrographic analysis was carried out on samples characterized by sandy mode fraction following the Indiana traditional method of point counting of 250 grains. A forward stepwise LDA was carried out using petrographic ratios and the mean grain-size $(\mathrm{Mz})$ based on the petrographic and textural data from KasperZubillaga and Dickinson (2001) (table 1). Also, an A-Nova test, post-hoc Tukey comparison tests and R-mode FA were carried out to assess the relations and differences among the compositional modal variables of the sands. The ratios were expressed as Log Qt/Ft (total quartz = mono and polycrystalline quartz/total feldspars = potash feldspars + plagioclase $)$, $\operatorname{LogQt} / \mathrm{Lt}$ (total quartz/total lithics $=$ volcanic + sedimentary), Log Ft/Lt (total feldspars/total lithics), Log Qm/Fk (monocrystalline quartz/potash feldspars), Log Qm/Fp (monocrystalline quartz/plagioclase), Log $\mathrm{Fk} / \mathrm{Fp}$ (potash feldspars/plagioclase) and Log Lv/Ls (volcanic lithics/sedimentary lithics) (table 2). This was done to avoid the constrained character of compositional data (Weltje, 2001). For the LDA, the variables were entered in a $N=33 \times n=8$ matrix, in which $N$ is the number of samples and $n$ is the number of variables. For the A-Nova tests, each column was labeled with each variable to test separately for $N=33$. For the R-mode FA, the samples and variables were



Figure 2. The major relief in the study area. The 200 and $1000 \mathrm{~m}$ contour lines are included to show the Tampico and Veracruz Embayments. Mountains, hills, and plain zones are adapted from Lugo-Hubp et al. (1990). Figura 2. Relieve principal del área de estudio. Se incluyeron las cotas de nivel de 200 y $1000 \mathrm{~m}$ para mostrar las cuencas de Tampico y veracruzana. Las montañas, colinas y planicies se adaptaron de Lugo-Hubp et al. (1990).

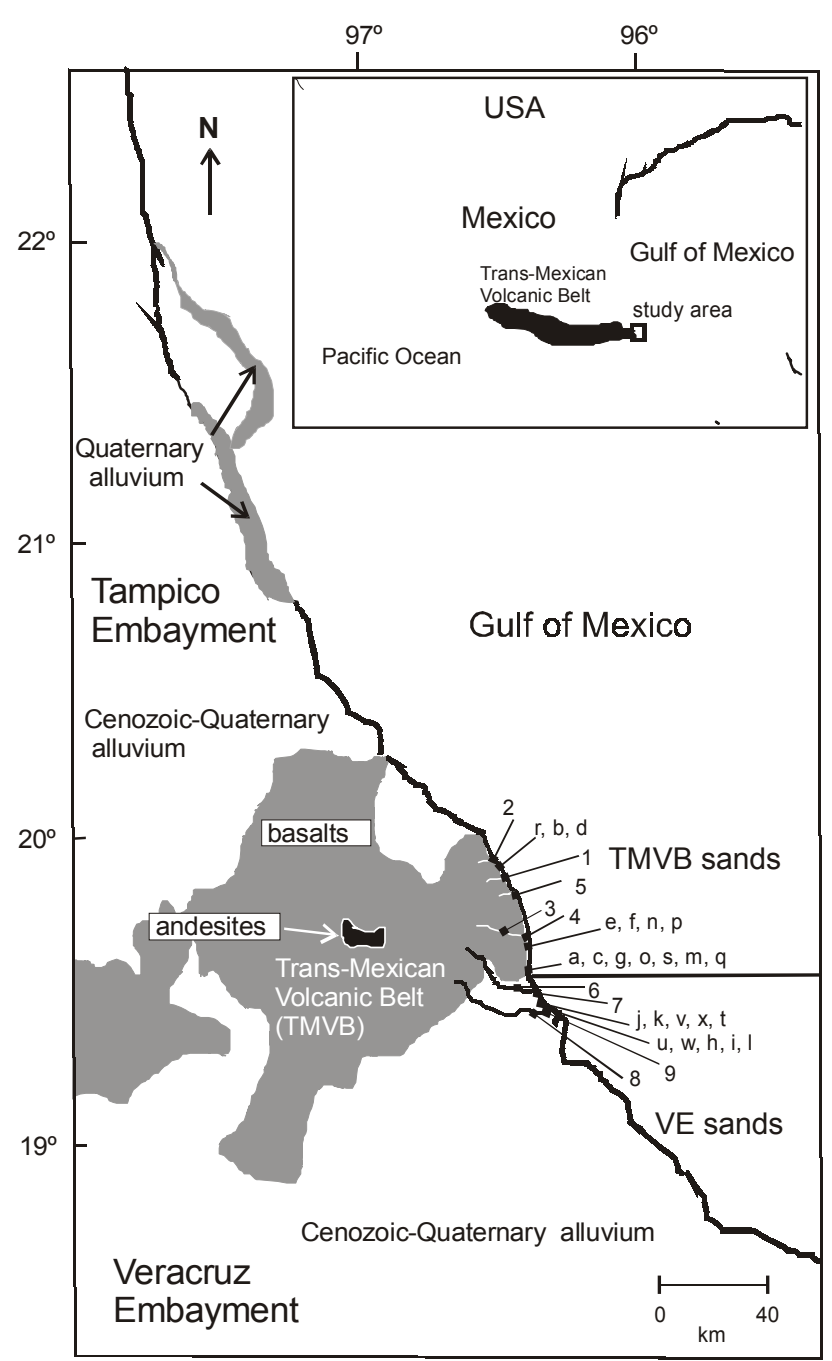

Figure 1. Geology and sampling sites of the study area.

Figura 1. Geología del área de estudio y ubicación de los sitios de muestreo

Placa de Cocos. La CT y la CV son depósitos sedimentarios compuestos de estratos aluviales del Terciario al Cuaternario de composición silicoclástica y, en menor medida, de carbonatos (fig. 1).

\section{Materiales y métodos}

Se recolectaron en total 33 muestras de sedimentos. El muestreo de arena se realizó únicamente en la temporada de secas donde las arenas fluviales de la CV todavía se encuentra drenando al mar (fig. 1). El análisis petrográfico se realizó en muestras que se caracterizaban por su fracción modal arenosa y de acuerdo al método tradicional Indiana de conteo puntual de 250 granos. Se realizó un ADL utilizando índices petrográficos y el tamaño medio de grano (Mz) con base en los datos petrográficos y texturales de Kasper-Zubillaga y Dickinson (2001) (tabla 1). También se realizaron una prueba A-Nova, una prueba de comparación Tukey post-hoc y un AF, con el fin de evaluar relaciones y diferencias entre las variables modales 


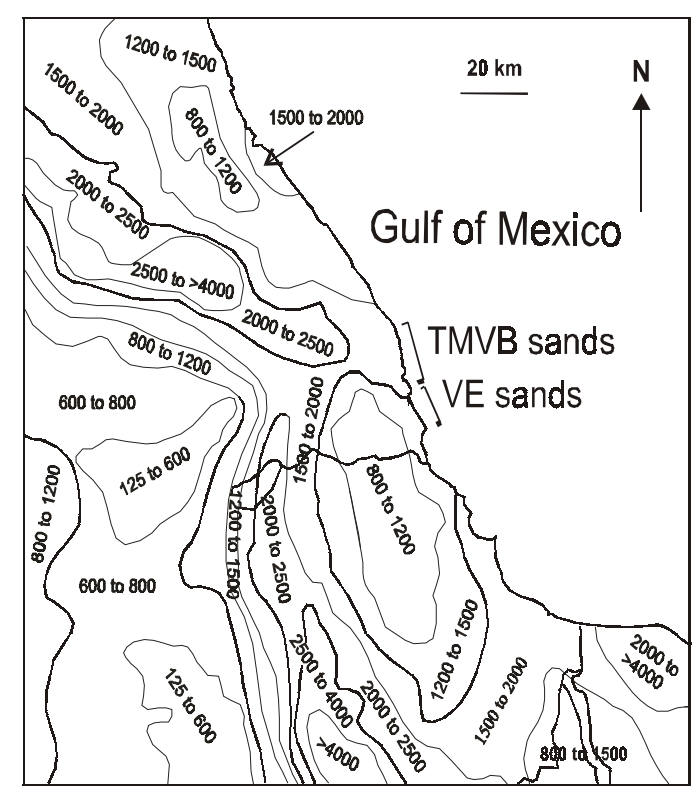

Figure 3. Average annual rainfall (modified from Vidal-Zepeda, 1990).

Figura 3. Precipitación media anual (modificada de Vidal-Zepeda, 1990).

grouped in a $N=19 \times n=8$ matrix for the beach, dune and river sands close to TMVB, and in a $N=14 \times n=8$ matrix for the southern beach, dune and river sands close to $\mathrm{VE}$ (figs. 1, 2). Hence, for the FA, samples a, b, c, d, e, f, g, m, n, o, $\mathrm{p}, \mathrm{q}, \mathrm{r}, \mathrm{s}, 1,2,3,4$ and 5 were grouped as TMVB sands in the FA correlation matrix, and samples h, i, j, k, 1, t, u, v, w, x, 6, 7, 8 and 9 were grouped as VE sands in the FA correlation matrix.

\section{Results}

The Qt-Ft-Lt ternary plots of beach, dune and river sands are shown in figure 4. Overall, the TMVB and VE sands are feldspatholithic $\left(\mathrm{Qt}_{30} \mathrm{Ft}_{24} \mathrm{Lt}_{46}\right)$. Furthermore, significant differ- composicionales de las arenas. Los índices fueron expresados como Log $\mathrm{Ct} / \mathrm{Ft}$ (cuarzo total = cuarzo mono y policristalino/ feldespato total $=$ feldespato potá-sico + plagioclasas), LogCt/ Lt $($ cuarzo total/líticos totales $=$ volcánicos + sedimentarios $)$, Log Ft/Lt (feldespato total/líticos totales), Log Cm/Fk (cuarzo monocristalino/feldespato potásico), Log $\mathrm{Cm} / \mathrm{Fp}$ (cuarzo monocristalino/plagioclasas), Log Fk/Fp (feldespato potásico/ plagioclasas) y Log Lv/Ls (líticos volcánicos/líticos sedimentarios) (tabla 2). Esto se hizo para evitar el carácter restringido de los datos composicionales (Weltje, 2001). Para el ADL las variables se pusieron en una matriz $N=33 \times n=8$, en la que $N$ es el número de muestras y $n$ el número de variables. Para las pruebas A-Nova, cada columna fue etiquetada con cada variable a probar por separado para $N=33$. Para el AF, muestras y variables fueron agrupadas en una matriz $N=19 \times n=8$ para las arenas de playa, duna y río cercanas al CVTM, y en una matriz $N=14 \times n=8$ para las arenas de playa, duna y río del sur, cercanas a la CV (figs. 1, 2). Por lo tanto, para el AF las muestras a, b, c, d, e, f, g, m, n, o, p, q, r, s, 1, 2, 3, 4 y 5 fueron agrupadas como arenas del CVTM en la matriz de correlación del AF, y las muestras h, i, j, k, 1, t, u, v, w, x, 6, 7, 8 y 9 lo fueron como arenas de la $\mathrm{CV}$.

\section{Resultados}

En la figura 4 se muestran los diagramas ternarios Ct-Ft-Lt de las arenas de playa, duna y río. Las arenas del CVTM y la $\mathrm{CV}$ son sobretodo feldespatolíticas $\left(\mathrm{Ct}_{30} \mathrm{Ft}_{24} \mathrm{Lt}_{46}\right)$. Además, se determinaron diferencias significativas, en porcentajes, entre la composición general de las arenas del CVTM y la CV (tabla 1). Esto se realizó mediante una prueba $t$ de una cola para Ct, Ft, Lt, Lv y Ls, en la que la hipótesis nula de igualdad de medias entre las arenas del CVTM y la CV es rechazada por las abundancias de cuarzo $\left(t_{\text {observada }}=2.09\right.$, g.1. $=31, \rho=0.05$, $\left.n=33, t_{\text {crítica }}=1.69\right)$. Adicionalmente, a partir de los valores parciales de lambda, los resultados del ADL muestran que los índices $\log \mathrm{Ct} / \mathrm{Ft}$, Log Ct/Lt, Log Ft/Lt y Log Lv/Ls son las
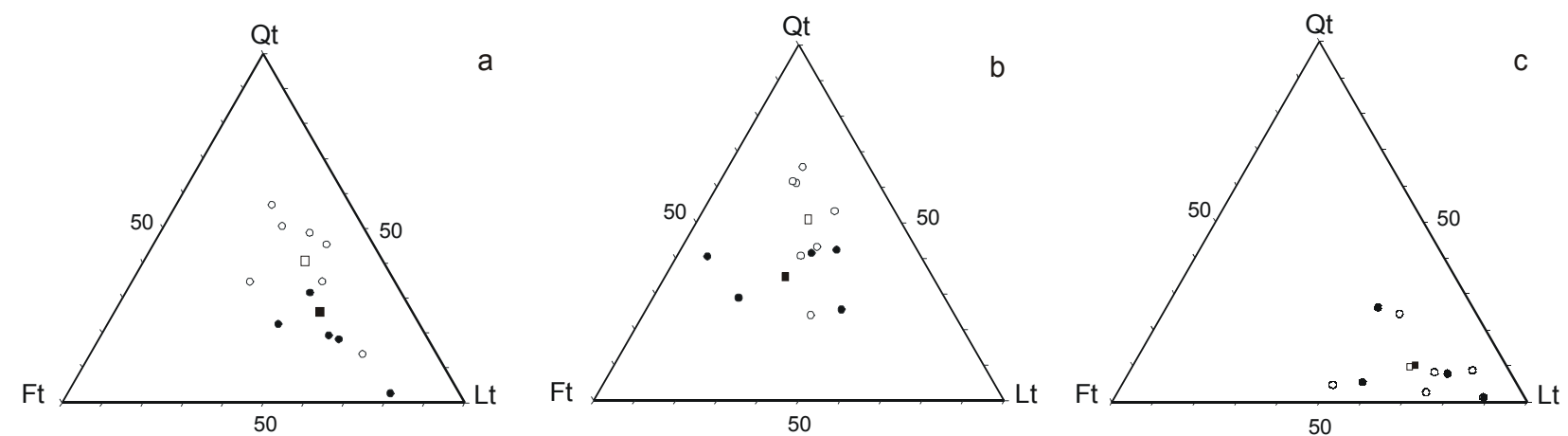

Figure 4. Qt-Ft-Lt ternary plots of $(\mathbf{a})$ beach $(n=12),(\mathbf{b})$ dune $(n=12)$ and $(\mathbf{c})$ river sands $(n=9)$. Trans-Mexican Volcanic Belt (TMVB) sands, -0-;

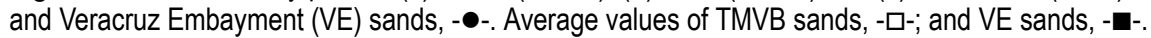

Figura 4. Diagramas ternarios Ct-Ft-Lt de las arenas de $(\mathbf{a})$ playa $(n=12),(\mathbf{b})$ duna $(n=12)$ y $(\mathbf{c})$ río $(n=9)$. Arenas del Cinturón Volcánico Trans-




Kasper-Zubillaga and Carranza-Edwards: Provenance of coastal sands in the Gulf of Mexico

Table 1. Petrographic data of beach, dune and river sands (\%). * Trans-Mexican Volcanic Belt sands, ** Veracruz Embayment sands.

Tabla 1. Datos petrográficos de las arenas de playa, duna y río (\%). *Arenas del Cinturón Volcánico Trans-Mexicano, **arenas de la Cuenca Veracruzana.

\begin{tabular}{|c|c|c|c|c|c|c|c|c|}
\hline Environment sample & Qt & Qm & $\mathrm{Ft}$ & $\mathrm{Fk}$ & $\mathrm{Fp}$ & $\mathrm{Lt}$ & $\mathrm{Lv}$ & Ls \\
\hline a Beach* & 45.4 & 67.1 & 11.6 & 4.4 & 28.5 & 43.0 & 83.0 & 17.0 \\
\hline b Beach* & 56.7 & 66.3 & 19.6 & 7.1 & 26.6 & 23.7 & 74.0 & 26.0 \\
\hline c Beach* & 48.7 & 62.0 & 14.2 & 12.7 & 25.5 & 37.1 & 72.0 & 28.0 \\
\hline d Beach* & 34.8 & 48.8 & 18.0 & 15.8 & 35.4 & 47.2 & 83.0 & 17.0 \\
\hline e Beach* & 14.0 & 16.1 & 18.4 & 1.8 & 82.1 & 67.6 & 91.0 & 9.0 \\
\hline f Beach* & 34.7 & 32.3 & 36.0 & 22.6 & 45.1 & 29.3 & 78.0 & 22.0 \\
\hline g Beach* & 50.7 & 52.3 & 20.0 & 5.8 & 34.9 & 29.3 & 70.0 & 30.0 \\
\hline Average & 40.7 & 49.8 & 19.7 & 10.1 & 40.1 & 39.6 & 75.6 & 24.4 \\
\hline h Beach** & 31.6 & 41.1 & 22.6 & 4.4 & 54.5 & 45.8 & 55.0 & 45.0 \\
\hline i Beach** & 18.2 & 22.2 & 22.2 & 1.4 & 76.4 & 59.6 & 75.0 & 25.0 \\
\hline j Beach** & 2.7 & 3.5 & 17.1 & 6.3 & 90.2 & 80.2 & 69.0 & 31.0 \\
\hline k Beach** & 19.3 & 21.7 & 24.1 & 5.2 & 73.1 & 56.6 & 70.0 & 30.0 \\
\hline 1 Beach** & 22.6 & 11.8 & 35.0 & 3.9 & 84.3 & 42.4 & 50.0 & 50.0 \\
\hline Average & 26.5 & 20.1 & 23.0 & 4.2 & 75.7 & 50.5 & 63.8 & 36.2 \\
\hline m Dune* & 53.2 & 70.0 & 14.7 & 3.2 & 26.8 & 32.1 & 77.0 & 23.0 \\
\hline n Dune* & 40.7 & 43.3 & 29.2 & 8.6 & 48.1 & 30.1 & 71.0 & 29.0 \\
\hline o Dune* & 61.1 & 68.5 & 20.1 & 6.5 & 25.0 & 18.8 & 77.0 & 23.0 \\
\hline p Dune* & 43.2 & 52.4 & 24.0 & 2.1 & 45.5 & 32.8 & 65.0 & 35.0 \\
\hline q Dune* & 24.0 & 25.3 & 35.1 & 0.9 & 73.8 & 40.9 & 90.0 & 10.0 \\
\hline r Dune* & 61.6 & 69.1 & 20.7 & 5.9 & 25.0 & 17.7 & 67.0 & 33.0 \\
\hline s Dune* & 65.6 & 74.8 & 16.3 & 10.8 & 14.4 & 18.1 & 77.0 & 23.0 \\
\hline Average & 49.9 & 57.7 & 22.9 & 5.4 & 36.9 & 27.2 & 74.8 & 25.2 \\
\hline t Dune** & 25.6 & 29.8 & 26.8 & 4.2 & 66.0 & 47.6 & 75.0 & 25.0 \\
\hline u Dune** & 28.9 & 29.8 & 50.3 & 0.8 & 69.4 & 20.8 & 75.0 & 25.0 \\
\hline v Dune** & 40.5 & 41.9 & 52.1 & 10.0 & 48.1 & 7.4 & 70.0 & 30.0 \\
\hline w Dune** & 41.5 & 48.7 & 26.2 & 4.3 & 47.0 & 32.3 & 75.0 & 25.0 \\
\hline x Dune** & 42.4 & 51.7 & 19.6 & 6.0 & 42.3 & 38.0 & 75.0 & 25.0 \\
\hline Average & 35.8 & 40.3 & 35.0 & 5.1 & 54.6 & 29.2 & 74.0 & 26.0 \\
\hline 1 River* & 2.7 & 2.9 & 23.0 & 6.8 & 90.3 & 74.3 & 97.0 & 3.00 \\
\hline 2 River* & 24.5 & 35.1 & 18.5 & 4.4 & 60.5 & 57.0 & 70.0 & 30.0 \\
\hline 3 River* & 4.8 & 2.8 & 44.4 & 9.4 & 87.8 & 50.8 & 91.0 & 9.0 \\
\hline 4 River* & 8.8 & 11.7 & 8.8 & 0.1 & 88.2 & 82.4 & 90.0 & 10.0 \\
\hline 5 River* & 8.3 & 8.9 & 18.2 & 4.3 & 86.8 & 73.5 & 94.0 & 6.0 \\
\hline Average & 9.8 & 12.3 & 22.6 & 5.0 & 82.7 & 67.6 & 88.4 & 11.6 \\
\hline 6 River** & 5.5 & 5.5 & 36.9 & 0.1 & 94.4 & 57.6 & 92.0 & 8.0 \\
\hline 7 River** & 7.9 & 10.4 & 15.3 & 0.1 & 89.5 & 76.8 & 97.0 & 3.0 \\
\hline 8 River** & 1.3 & 1.3 & 9.9 & 7.9 & 90.8 & 88.8 & 90.0 & 10.0 \\
\hline 9 River** & 26.3 & 32.1 & 22.8 & 4.2 & 63.7 & 50.9 & 90.0 & 10.0 \\
\hline Average & 10.3 & 12.3 & 21.2 & 3.1 & 84.6 & 68.5 & 92.2 & 7.8 \\
\hline
\end{tabular}

$\mathrm{Qt}=$ total quartz = mono + polycrystalline quartz; $\mathrm{Ft}=$ total feldspars = potash feldspars + plagioclase; $\mathrm{Lt}=$ total lithics = volcanic + sedimentary lithics; $\mathrm{Qm}=$ monocrystalline quartz; Fk = potash feldpars; Fp = Ca-Na plagioclase; Lv = volcanic lithics (andesites, basalts); Ls = sedimentary lithics (beachrock, sandstones, siltstones, chert). 
ences between the overall composition of the TMVB and VE sands, in percent values (table 1), were determined. This was carried out with a one-tailed $t$-test for Qt, Ft, Lt, Lv and Ls, in which the null-hypothesis of equal means is rejected between the TMVB and VE sands for quartz abundances $\left(t_{\text {observed }}=2.09\right.$, $\left.\mathrm{df}=31, \rho=0.05, n=33, t_{\text {critical }}=1.69\right)$. Additionally, the LDA results show, from the partial lambda values, that the best variables that discriminate between the TMVB and VE sands are the $\log \mathrm{Qt} / \mathrm{Ft}, \log \mathrm{Qt} / \mathrm{Lt}, \log \mathrm{Ft} / \mathrm{Lt}$ and $\mathrm{Log} \mathrm{Lv} / \mathrm{Ls}$ ratios (table 3). Moreover, the best canonical score plots were between canonical variate $1 \mathrm{vs}$ canonical variate 2 (root $1 \mathrm{vs}$ root 2) and canonical variate $2 v s$ canonical variate 3 for the dune and river TMVB and VE sands (figs. 5, 6). The best separated groups were the TMVB river sands distinct from the VE river sands, followed by the TMVB beach sands distinct from the VE beach sands, and the TMVB dune sands distinct from the VE dune sands (fig. 5). Discrimination between the TMVB and VE beach, dune and river sands is controlled by the Log Qt/Ft, Log Qt/Lt, Log Ft/Lt and Log Lv/Ls ratios. However, a comparison of means test may elucidate the contribution of the variable in terms of separate groups. Therefore, an A-Nova test and post-hoc Tukey comparison of means test were carried out, and we found that the TMVB and VE dune and river sands are significantly different when applying these tests (table 4). Moreover, factor 1 grouped the Log Qt/Ft, Log Qt/Lt, Log Qm/Fk, Log Qm/Fp and Log Lv/Ls ratios, and Mz as high variable loads for the TMVB sands (table 5). Additionally, factor 1 grouped the Log Qt/Ft, Log Qt/Lt, Log Ft/Lt, Log $\mathrm{Qm} / \mathrm{Fp}$ and Log Lv/Ls ratios for the VE sands. This suggests that the composition of the TMVB beach, dune and river sands is controlled by the Log Qt/Ft, Log Qt/Lt, Log Qm/Fk, Log $\mathrm{Qm} / \mathrm{Fp}$ and Log Lv/Ls ratios, as well as $\mathrm{Mz}$, and that the VE sand composition is controlled by the $\log \mathrm{Qt} / \mathrm{Ft}, \log \mathrm{Qt} / \mathrm{Lt}$,

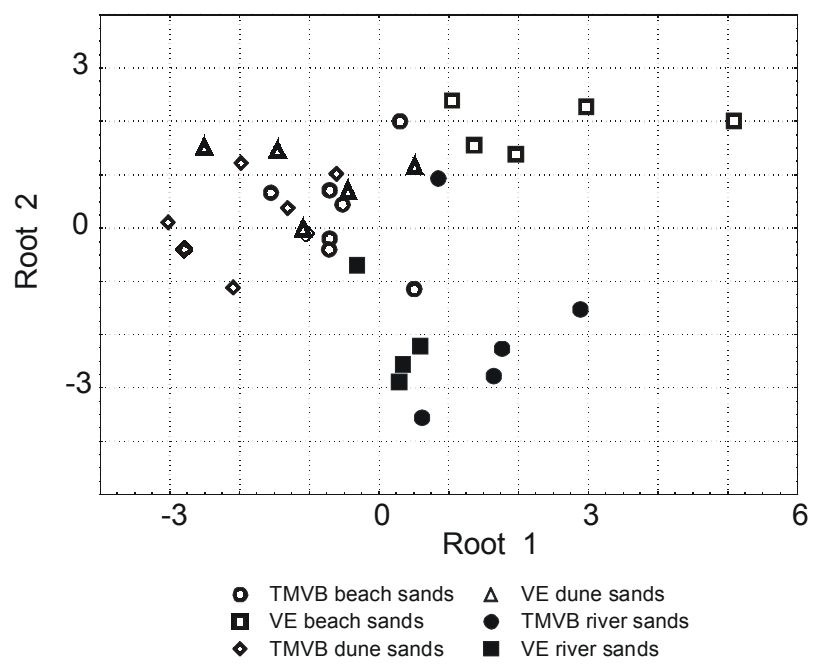

Figure 5. Canonical variate diagram for discriminant function scores $(n=33)$ : root 1 vs root 2 .

Figura 5. Diagrama de variables canónicas para los valores de la función discriminante $(n=33)$ : raíz 1 vs. raíz 2 variables que mejor discriminan entre las arenas del CVTM y la CV (tabla 3). Además, los mejores diagramas de valores canónicos para las arenas de duna y río del CVTM y la CV (figs. 5, 6) se dieron entre las variables canónicas 1 y 2 (raíz 1 vs. raíz 2) y entre las variables canónicas 2 y 3 para las arenas de duna y río del CVTM y la CV. Los grupos mejor separados fueron las arenas de río del CVTM distintas de las arenas de río de la CV, seguidos por las arenas de playa del CVTM distintas de las arenas de playa de la CV y las arenas de duna del CVTM distintas de las arenas de duna de la CV (fig. 5). La discriminación entre las arenas de playa, duna y río del CVTM y la CV se encuentra controlada por los índices $\log \mathrm{Ct} / \mathrm{Ft}$, Log Ct/Lt, Log Ft/Lt y Log Lv/Ls. Sin embargo, una prueba de comparación de medias puede dilucidar la contribución de cada variable en términos de los distintos grupos. Por lo tanto, se realizaron una prueba A-Nova y una comparación Tukey post-hoc de medias $\mathrm{y}$, al aplicarse estas pruebas, se encontró que las arenas de duna y de río del CVTM y la CV son significativamente diferentes (tabla 4). Por otra parte, el factor 1 agrupó los índices Log Ct/ $\mathrm{Ft}, \log \mathrm{Ct} / \mathrm{Lt}, \log \mathrm{Cm} / \mathrm{Fk}, \log \mathrm{Cm} / \mathrm{Fp}, \log \mathrm{Lv} / \mathrm{Ls}$ y a $\mathrm{Mz}$ como cargas altamente variables para las arenas del CVTM (tabla 5). Además, el factor 1 agrupó los índices $\log \mathrm{Ct} / \mathrm{Ft}$, $\log \mathrm{Ct} / \mathrm{Lt}, \log \mathrm{Ft} / \mathrm{Lt}, \log \mathrm{Cm} / \mathrm{Fp}$ y Log Lv/Ls de las arenas de la $\mathrm{CV}$. Esto sugiere que la composición de las arenas de playa, duna y río del CVTM está controlada por los índices $\log \mathrm{Ct} / \mathrm{Ft}$, $\log \mathrm{Ct} / \mathrm{Lt}$, Log Cm/Fk, Log Cm/Fp y Log Lv/Ls, así como por $\mathrm{Mz}$, y que la composición de las arenas de la CV está controlada por los índices $\log \mathrm{Ct} / \mathrm{Ft}$, Log $\mathrm{Ct} / \mathrm{Lt}, \log \mathrm{Ft} / \mathrm{Lt}$, Log Cm/ Fp y Log Lv/Ls (tabla 5). Sin embargo, los índices Log $\mathrm{Ct} / \mathrm{Ft}$, $\log \mathrm{Ct} / \mathrm{Lt}$ y Log Lv/Ls son las únicas tres variables significativas incluidas en el ADL que contribuyen a diferenciar entre las arenas de duna y río del CVTM y la CV. Estos resultados se basan en la significancia estadística general de las pruebas de

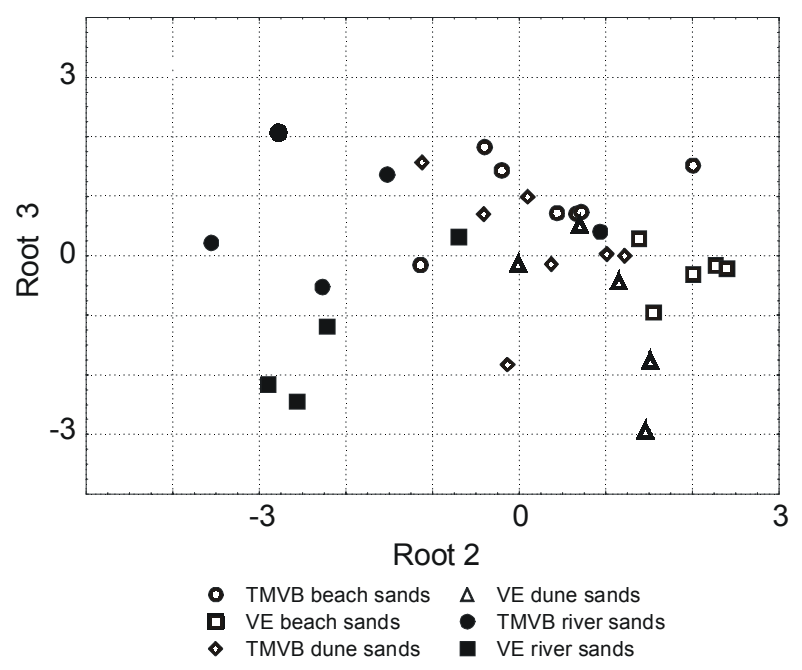

Figure 6. Canonical variate diagram for discriminant function scores $(n=33)$ : root 2 vs root 3.

Figura 6. Diagrama de variables canónicas para los valores de la función discriminante $(n=33)$ : raíz 2 vs. raíz 3 
Table 2. Compositional data tranformation to Log ratios. ${ }^{*}$ Trans-Mexican Volcanic Belt sands, ${ }^{* *}$ Veracruz Embayment sands.

Tabla 2. Transformación de los datos composicionales a índices logarítmicos. *Arenas del Cinturón Volcánico Trans-Mexicano, **arenas de la Cuenca Veracruzana.

\begin{tabular}{|c|c|c|c|c|c|c|c|}
\hline Samples & $\log \mathrm{Qt} / \mathrm{Ft}$ & $\log \mathrm{Qt} / \mathrm{Lt}$ & Log Ft/Lt & LogQm/Fk & $\log \mathrm{Qm} / \mathrm{Fp}$ & Log Fk/Fp & $\log \mathrm{Lv} / \mathrm{Ls}$ \\
\hline a Beach* & 0.59 & 0.02 & -0.59 & 1.18 & 0.37 & -0.82 & 0.69 \\
\hline b Beach* & 0.46 & 0.38 & -0.09 & 0.97 & 0.40 & -0.59 & 0.45 \\
\hline c Beach* & 0.53 & 0.12 & -0.42 & 0.69 & 0.39 & -0.30 & 0.00 \\
\hline d Beach* & 0.29 & -0.14 & -0.42 & 0.49 & 0.14 & -0.36 & 0.69 \\
\hline e Beach* & -0.12 & -0.70 & -0.57 & 0.95 & -0.72 & -1.70 & 1.00 \\
\hline f Beach* & -0.02 & 0.07 & 0.09 & 0.15 & -0.15 & -0.30 & 0.55 \\
\hline g Beach* & 0.40 & 0.24 & -0.17 & 0.95 & 0.17 & -0.80 & 0.37 \\
\hline Average & 0.30 & 0.00 & -0.31 & 0.77 & 0.09 & -0.69 & 0.54 \\
\hline h Beach** & 0.15 & -0.16 & -0.31 & 0.98 & -0.12 & -1.10 & 0.09 \\
\hline i Beach** & -0.09 & -0.52 & -0.43 & 1.20 & -0.54 & -2.00 & 0.48 \\
\hline j Beach** & -0.82 & -1.52 & -0.68 & -0.27 & -1.52 & -1.15 & 0.35 \\
\hline k Beach** & -0.10 & -0.47 & -0.38 & 0.62 & -0.54 & -1.15 & 0.37 \\
\hline $1 \mathrm{Beach}^{* *}$ & -0.19 & -0.28 & -0.09 & 0.48 & -0.89 & -1.40 & 0.00 \\
\hline Average & -0.21 & -0.59 & -0.38 & 0.60 & -0.72 & -1.36 & 0.26 \\
\hline m Dune* & 0.56 & 0.22 & -0.35 & 1.34 & 0.42 & -0.96 & 0.53 \\
\hline n Dune* & 0.14 & 0.13 & -0.01 & 0.70 & -0.05 & -0.77 & 0.39 \\
\hline o Dune* & 0.48 & 0.51 & 0.03 & 1.02 & 0.44 & -0.59 & 0.51 \\
\hline p Dune* & 0.26 & 0.12 & -0.14 & 1.40 & 0.06 & -1.40 & 0.27 \\
\hline q Dune* & -0.17 & -0.24 & -0.07 & 1.45 & -0.47 & -2.00 & 0.95 \\
\hline r Dune* & 0.47 & 0.54 & 0.06 & 1.07 & 0.44 & -0.64 & 0.31 \\
\hline s Dune* & 0.60 & 0.56 & -0.05 & 0.84 & 0.72 & -0.12 & 0.52 \\
\hline Average & 0.34 & 0.26 & -0.07 & 1.12 & 0.22 & -0.92 & 0.50 \\
\hline t Dune** & -0.02 & -0.28 & -0.25 & 0.85 & -0.35 & -1.22 & 0.48 \\
\hline u Dune** & -0.24 & 0.14 & 0.38 & 1.57 & -0.38 & -2.00 & 0.48 \\
\hline v Dune** & -0.11 & 0.74 & 0.85 & 0.62 & -0.06 & -0.70 & 0.37 \\
\hline w Dune** & 0.20 & 0.11 & -0.09 & 1.05 & 0.01 & -0.05 & 0.48 \\
\hline x Dune** & 0.33 & 0.05 & -0.29 & 0.94 & 0.09 & -0.85 & 0.48 \\
\hline Average & 0.03 & 0.15 & 0.12 & 1.01 & -0.14 & -0.96 & 0.46 \\
\hline 1 River* & -0.96 & -1.52 & -0.52 & -0.38 & -1.52 & -1.15 & 1.51 \\
\hline 2 River* & 0.12 & -0.38 & -0.49 & 0.90 & -0.24 & -1.15 & 0.37 \\
\hline 3 River* & -1.00 & -1.05 & -0.06 & -0.54 & -1.52 & -1.00 & 1.00 \\
\hline 4 River* & 0.00 & -1.00 & -1.00 & 2.07 & -0.89 & -1.00 & 0.95 \\
\hline 5 River* & -0.35 & -0.96 & -0.62 & 0.31 & -1.00 & -1.40 & 1.19 \\
\hline Average & -0.44 & -0.98 & -0.54 & 0.47 & -1.03 & -1.14 & 1.01 \\
\hline 6 River** & -0.85 & -1.05 & -0.19 & 1.74 & -1.30 & -1.00 & 1.06 \\
\hline 7 River** & -0.29 & -1.00 & -0.72 & 2.02 & -0.96 & -3.00 & 1.51 \\
\hline 8 River** & -0.89 & -2.00 & -0.96 & -0.80 & -2.00 & -1.10 & 0.95 \\
\hline 9 River** & 0.06 & -0.29 & -0.36 & 0.88 & -0.30 & -1.22 & 0.95 \\
\hline Average & -0.49 & -1.08 & -0.56 & 0.96 & -1.14 & -1.58 & 1.12 \\
\hline
\end{tabular}

See table 1 for symbols. 
Table 3. Summary of the linear discriminant analysis (LDA). Partial lambda values in bold are the most significant values in the LDA.

Tabla 3. Resumen del análisis de discriminación lineal (ADL). Los valores parciales de lambda más significativos del ADL se muestran en negritas.

\begin{tabular}{lccccc}
\hline Variables & Wilks' lambda & Partial lambda & F-remove & p-level & Tolerance \\
\hline Log Qt/Ft & 0.06 & $\mathbf{0 . 5 3}$ & 3.47 & 0.02 & 0.99 \\
Log Qt/Lt & 0.06 & $\mathbf{0 . 5 3}$ & 3.42 & 0.02 & 0.99 \\
Log Ft/Lt & 0.06 & $\mathbf{0 . 5 1}$ & 3.77 & 0.01 & 0.99 \\
Log Qm/Fk & 0.04 & 0.73 & 1.45 & 0.24 & 0.65 \\
Log Qm/Fp & 0.03 & 0.90 & 0.43 & 0.81 & 0.95 \\
Log Fk/Fp & 0.04 & 0.83 & 0.77 & 0.58 & 0.49 \\
Log Lv/Ls & 0.07 & $\mathbf{0 . 4 5}$ & 4.71 & 0.00 & 0.48 \\
Mz & 0.04 & 0.70 & 1.71 & 0.17 & 0.30 \\
\hline
\end{tabular}

See table 1 for symbols.

Table 4. A-Nova tests and post-hoc values of variables and groups in the linear discriminant analysis: Trans-Mexican Volcanic Belt (TMVB) and Veracruz Embayment (VE). Most significant post-hoc values and MS effect are in bold.

Tabla 4. Valores de las pruebas A-Nova y post hoc de variables y grupos del análisis de discriminación lineal: Cinturón Volcánico TransMexicano (TMVB) y Cuenca Veracruzana (VE). Los valores más significativos de la prueba post-hoc y del efecto MS se muestran en negritas.

\begin{tabular}{lcccccc}
\hline Variable & MS effect & MS error & F & $\rho$ & $\begin{array}{l}\text { TMVB-VE } \\
\text { dune sands }\end{array}$ & $\begin{array}{c}\text { TMVB-VE } \\
\text { river sands }\end{array}$ \\
\hline Log Qt/Ft & $\mathbf{0 . 7 1}$ & 0.12 & 5.70 & $<0.05$ & $\mathbf{0 . 0 6}$ & $\mathbf{0 . 0 6}$ \\
Log Qt/Lt & $\mathbf{1 . 8 1}$ & 0.18 & 9.60 & $<0.05$ & 0.99 & 0.99 \\
Log Ft/Lt & 0.35 & 0.09 & 3.93 & $<0.05$ & $\mathbf{0 . 0 6}$ & 0.99 \\
LogLv/Ls & $\mathbf{0 . 5 3}$ & 0.07 & 7.34 & $<0.05$ & 0.99 & $\mathbf{0 . 0 0}$ \\
\hline
\end{tabular}

$\log \mathrm{Ft} / \mathrm{Lt}, \log \mathrm{Qm} / \mathrm{Fp}$ and $\log \mathrm{Lv} / \mathrm{Ls}$ ratios (table 5). Nevertheless, only the Log Qt/Ft, Log Qt/Lt and Log Lv/Ls ratios are the three significant variables entered in the LDA that contribute to separate among TMVB and VE dune and river sands. These results are based on the overall statistical significance of the LDA, A-Nova, post-hoc and FA tests for the three most common significant variables entered in the analyses (i.e., Log Qt/Ft, Log Qt/Lt and $\log \mathrm{Lv} / \mathrm{Ls}$ ratios) (tables 3, 4, 5).

\section{Discussion}

It can be observed that dune sands tend to concentrate towards the Qt pole, whereas the beach and river sands tend to concentrate towards the Lt pole (fig. 4). This suggests a quartzrich trend for the dune sands. Moreover, the $t$-test results of null-hypothesis rejection suggest that the overall quartz abundances of the sand samples from TMVB and VE are significantly different. Additionally, the LDA, A-Nova and FA results showed that the Miocene-Pliocene andesites and basalts control the VE river sand composition. This is supported by the highest concentration of $\mathrm{Lv}$ in the $\mathrm{VE}$ river sands (a more positive $\log \mathrm{Lv} / \mathrm{Ls}$ ratio than the $\mathrm{Log} \mathrm{Lv} / \mathrm{Ls}$ ratio from the TMVB river sands) (tables 1,2), which suggests: (a) that abrasion of Ls is occurring throughout the long VE rivers due to the longer
ADL, A-Nova, AF y post-hoc para las tres variables significativas más comunes incluidas en los análisis (i.e., los índices $\log \mathrm{Ct} / \mathrm{Ft}$, Log Ct/Lt y Log Lv/Ls) (tablas 3, 4, 5).

\section{Discusión}

Se puede observar que las arenas de duna tienden a concentrarse hacia el vértice $\mathrm{Ct}$ mientras que las arenas de playa y río tienden a hacerlo hacia Lt (fig. 4), lo que sugiere un enriquecimiento en cuarzo en las arena de duna. Por otra parte, el rechazo de la hipótesis nula resultante de la prueba $t$ sugiere que las abundancias generales de cuarzo de las muestras de arena del CVTM y la CV son significativamente diferentes. Además, los resultados del ADL, el A-Nova y el AF mostraron que las andesitas y los basaltos del Plioceno-Mioceno controlan la composición de las arenas de río de la CV. Esto se sustenta en la mayor concentración de Lv encontrada en la arena de río de la $\mathrm{CV}$ (un índice Log Lv/Ls más positivo que para la arena de río del CVTM) (tablas 1,2), lo que sugiere: (a) que la abrasión de Ls está sucediendo a todo lo largo de los ríos de la CV debido a la más prolongada intemperización químico/mecánica de los Ls de la arena de río de la CV (Arribas et al., 2000); y/o (b) que los ríos de la CV están muy influenciados por el aporte del CVTM en sus cabeceras, permitiendo una 
chemical/mechanical weathering acting upon the Ls in the VE river sands (Arribas et al., 2000); and/or (b) that VE rivers are highly influenced by the TMVB supply at their heads, giving the VE river sands minimal maturation despite the long detritus transport (Le Pera et al., 2001) (fig. 1; tables 1, 2). The $\log \mathrm{Qt} / \mathrm{Lt}$ ratio also controls the compositional differences between the TMVB dune sands and VE dune sands. A quartz supply derived from TE by a longshore drift southwards may be contributing to the composition of the TMVB dune sands by effect of marine/aeolian selective transport that enriches the dune sands with more quartz in the TMVB dune sands compared to the VE dune sands (Kasper-Zubillaga and Dickinson, 2001; Frihy et al., 1999) (table 2). This quartz enrichment effect in the dune may also be related to depletion of labile grains in humid climatic conditions (Mack, 1984). Hence, the major compositional control of the TMVB dune and VE river sands is based on the quartz content of the dune and the volcanic lithic content in the river.

Multivariate procedures showed that the Log Qt/Ft and Log $\mathrm{Qt} / \mathrm{Lt}$ ratios and the Log Lv/Ls ratio are the three significant variables entered in the LDA that contribute to separate among TMVB and VE dune and river sands. Moreover, Log Qt/Ft and Log Lv/Ls are the two most important variables with geological significance to discriminate between TMVB and VE dune sands and TMVB and EV river sands. TMVB dune sand and VE river sand composition is controlled by a longshore drift of quartz supply, depletion of Ls by mechanical and/or chemical weathering and the influence of TMVB on the VE river sand composition. Multivariate procedures are a useful tool to elucidate the relationship between source rocks and sediments.

\section{Acknowledgements}

Financial support was provided by CONACYT (Mexico's Council of Science and Technology, project 3477T). Reviews by E. Le Pera and S. Critelli are highly appreciated. We thank Eduardo Morales de la Garza for his collaboration with the drawings.

\section{References}

Arribas, J., Critelli, S., Le Pera, E. and Tortosa, A. (2000). Composition of modern stream sand derived from a mixture of sedimentary and metamorphic source rocks (Henares River, Central Spain). Sedimen. Geol., 133: 27-48.

Basu, A. (1976) Petrology of Holocene fluvial sand derived from plutonic source rocks: Implications for paleoclimatic interpretation. J. Sediment. Petrol., 46: 694-709.

Cantagrel, J.M. and Robin, C. (1979). K-Ar dating in eastern volcanic rocks - relations between the andesite and the alkaline provinces. J. Volc. Geoth. Res., 5: 99-114.

CETENAL (1970). Carta de Climas (Veracruz 14Q-VI). Scale 1:500,000. Secretaría de la Presidencia and Instituto de Geografía, México.

Critelli, S., Le Pera, E. and Ingersoll, R.V. (1997). The effects of source lithology, transport, deposition and sampling scale on the composition of southern California sand. Sedimentology, 44: 653-671.
Table 5. Factor analysis of Trans-Mexican Volcanic Belt (TMVB) and Veracruz Embayment (VE) beach, dune and river sand data. The highest values of the variables for each factor are in bold.

Tabla 5. Análisis factorial de los datos de las arenas de playa, duna y río del Cinturón Volcánico Trans-Mexicano (TMVB) y la Cuenca Veracruzana (VE). Los valores más altos de las variables para cada factor se muestran en negritas.

\begin{tabular}{lcccccc}
\hline & \multicolumn{2}{c}{ TMVB sands } & & \multicolumn{2}{c}{ VE sands } \\
\cline { 2 - 3 } \cline { 6 - 7 } & F1 & F2 & & F1 & F2 \\
\hline Log Qt/Ft & $\mathbf{0 . 9 0}$ & 0.35 & & $\mathbf{0 . 8 9}$ & 0.01 \\
Log Qt/Lt & $\mathbf{0 . 6 9}$ & $\mathbf{0 . 6 8}$ & & $\mathbf{0 . 9 7}$ & 0.05 \\
Log Ft/Lt & 0.04 & $\mathbf{0 . 8 2}$ & & $\mathbf{0 . 7 0}$ & 0.10 \\
Log Qm/Fk & $\mathbf{0 . 8 7}$ & -0.37 & & 0.46 & $\mathbf{- 0 . 8 0}$ \\
Log Qm/Fp & $\mathbf{0 . 7 8}$ & 0.56 & & $\mathbf{0 . 9 7}$ & 0.00 \\
Log Fk/Fp & 0.39 & $\mathbf{0 . 7 3}$ & & 0.17 & $\mathbf{0 . 7 6}$ \\
Log Lv/Ls & $\mathbf{- 0 . 6 6}$ & -0.58 & & $-\mathbf{0 . 7 3}$ & -0.43 \\
Mz & $\mathbf{0 . 6 6}$ & 0.12 & & 0.76 & 0.23 \\
Prop. Tot. & 0.45 & 0.32 & & 0.52 & 0.23 \\
\hline
\end{tabular}

mínima maduración de la arena de río de la $\mathrm{CV}$ a pesar del largo transporte de detritos (Le Pera et al., 2001) (fig. 1; tablas 1, 2). El índice Log Ct/Lt también controla la diferencia de composición entre las arenas de duna del CVTM y las de la CV. El aporte de cuarzo transportado hacia el sur a lo largo de la costa desde la Cuenca Tampico podría estar contribuyendo en la composición de las arenas de duna del CVTM por efecto de un transporte selectivo eólico/marino que enriquece con cuarzo las arenas de duna del CVTM en comparación con las arenas de duna de la CV (Kasper-Zubillaga y Dickinson, 2001; Frihy et al., 1999) (tabla 2). Este efecto de enriquecimiento en cuarzo en las dunas también podría estar relacionado con la ausencia de granos lábiles en condiciones climáticas húmedas (Mack, 1984). Por lo tanto, el control composicional de las arenas de duna del CVTM y de río de la CV lo ejerce básicamente el contenido de cuarzo de la duna y el contenido lítico volcánico del río.

Los métodos multivariados mostraron que los índices Log $\mathrm{Ct} / \mathrm{Ft}, \log \mathrm{Ct} / \mathrm{Lt}$ y $\log \mathrm{Lv} / \mathrm{Ls}$ son las tres variables significativas incluidas en el ADL que contribuyen a discernir entre las arenas de duna y río del CVTM y la CV. Además Log Ct/Ft y Log Lv/Ls son las dos variables más importantes con significancia geológica para discriminar entre las arenas de duna del CVTM y la CV, y las arenas de río del CVTM y la CV. Las composiciones de la arena de duna del CVTM y de la arena de río de la CV están controladas por el transporte a lo largo de la costa del aporte de cuarzo, por la degradación de Ls por intemperización mecánica y/o química y por la influencia del CVTM en la composición de la arena de río de la CV. Los métodos de análisis multivariado constituyen una herramienta útil para dilucidar la relación entre la roca fuente y los sedimentos. 
Fernández-Eguiarte, A., Gallegos-García, A. y Zavala-Hidalgo, J., (1992a). Oceanografía Física 1: Masas de Agua y Mareas de los Mares Mexicanos. Atlas Nacional de México (Sheet IV.9.1, summer). Instituto de Geografía, UNAM, México.

Fernández-Eguiarte, A., Gallegos-García, A. y Zavala-Hidalgo, J., (1992b). Oceanografía Física 1: Masas de Agua y Mareas de los Mares Mexicanos. Atlas Nacional de México (Sheet IV.9.2, winter). Instituto de Geografía, UNAM, México.

Frihy, O.E., El-Askary, M.A., Deghidy, E.M. and Moufaddal, W.M. (1999). Differentiating fluvio-marine depositional environments in the Nile Delta using textural and compositional components. J. Afr. Earth Sci., 28: 599-618.

Ibbeken, H. and Schleyer, R. (1991) Source and Sediment. Springer Verlag, Berlin, 286 pp.

Kasper-Zubillaga, J.J. and Dickinson, W.W. (2001). Discriminating depositional environments from modern source terranes using modal analysis. Sediment. Geol., 143: 149-167.

Komar, P.D. (1976). Beach Processes and Sedimentation. Prentice Hall, New Jersey, 429 pp.

Le Pera, E. and Critelli, S. (1997) Sourceland controls on the composition of beach and fluvial sand of the northern Tyrrenian coast of Calabria, Italy: Implications for actualistic petrofacies. Sediment. Geol., 110: 81-97.

Le Pera, E., Arribas, J., Critelli, S. and Tortosa, A. (2001) The effects of source rocks and chemical weathering on the petrogenesis of siliciclastic sand from the Neto River (Calabria, Italy): Implications for provenance studies. Sedimentology, 48: 357-378.

Lugo-Hubp, J., Aceves-Quezada, F., Córdoba Fernández de Arteaga, C., Espinasa-Pereña, R., García-Arizaga, M.T. y Melo-Gallegos, C. (1990). El Relieve como Atractivo Natural. Estado de los

\section{Agradecimientos}

Esta investigación fue financiada por CONACYT, proyecto 3477T. Se agradecen las revisiones hechas por E. Le Pera y S. Critelli. Agradecemos a Eduardo Morales de la Garza su colaboración con las figuras.

Traducido al español por Manuel Gardea-Ojeda.

Componentes Naturales del Medio Ambiente, Hoja V.2.1, scale 1:4000000. Atlas Nacional de México, Instituto de Geografía, Univ. Nal. Autón. México.

Mack, G.E. (1984). Exceptions to the relationship between plate tectonics and sandstone composition. J. Sedimen. Petrol., 54: 212-220.

Negendank, J.F., Emmermann, R., Krawczyk, R., Mooser, F., Tobschall, H. and Werle, D. (1985) Geological and geochemical investigation on the eastern Trans-Mexican Volcanic Belt. Geofís. Int., 24: 477-575.

Vidal-Zepeda, R. (1990). Precipitación: Clima, Hoja IV.4.6, scale 1:4000000. Atlas Nacional de México. Instituto de Geografía, Univ. Nal. Autón. México.

Weltje, G.J. (2001) Quantitative analysis of detrital modes: Statistically rigorous confidence regions in ternary diagrams and their use in sedimentary petrology. Earth Sci. Rev., 57: 211-253. 